\title{
交感神經末梢降压葯物化学葯理的进展
}

\author{
白东魯
}

心血管系梳疾患是危害人类健康最严重 的疾病之一, 其中尤以原发性高血压的发病 率和死亡率为最高。长时期来, 人們为了征 服它，作了很大努力，但进展不大。朁未能 最終關明其病因, 又未能在外科手术和物理 治疗上找到䨍意的方法。在䒺物研究方面, 虽也找到了数种能用于临床，并具有一定疗 效的降压䓎, 但大多缺乏稳定持久的降压效 果，持存在各种各样副作用。最近五年来， 由于葯化学家、䒺理学家、生化学家和临床 医生的共同努力，在高血压病药物治疗上取 得了丰碩的成果。确証了儿茶酚胺体內合成 和代謝的主要途径。根据以前积累和新近发 現的資料，提出了降压药物作用机制的新覌 点。現有䒺物如利血平、胍乙啶、溴茮乙銨 和二氢氯噻嗪等, 已使医生在絕大多数情况 下,有可能有效地控制病人血压、改善症状， 使高血压病死亡率有了下降。

本交拟从下列三方面对作用于交感神經 末梢降压葯物化学䒺理的进展作一介紹:

1. 儿茶酚胺的生物合成和代謝;

2. 作用于交感神經末梢和影响儿茶酸胺 合成和代謝的几类降压䒺物作用机制及化学 結构与䒺理作用关系;

3. 腎上腺素能受体。

\section{一 儿茶酚胺的生物合成和代謝}

为了解决高血压病发病机轉和降压葯物 作用历程中的一係列問題，我們应具有儿茶 酚胺体內形成、轉化的丰富的生化知識。这 方面的主要問題目前已了解得相当清楚了, 但仍留下許多疑点有待进一步研究探索。

早在 1939 年已有人提出了儿茶酚胺生 物合成途径, 但由于当时技术上的限制, 各 人所得結果既不肯定又互相矛盾。近十余年 来同位素标記、光謭等新技术和其它微量組 織化学方法的发展和广泛应用, 使这方面工 作取得很大进展。它們生物合成的主要途径 可表示如下[1]:

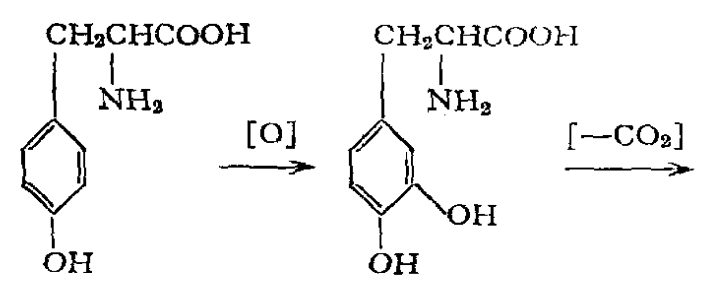

1

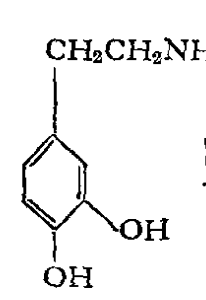

III

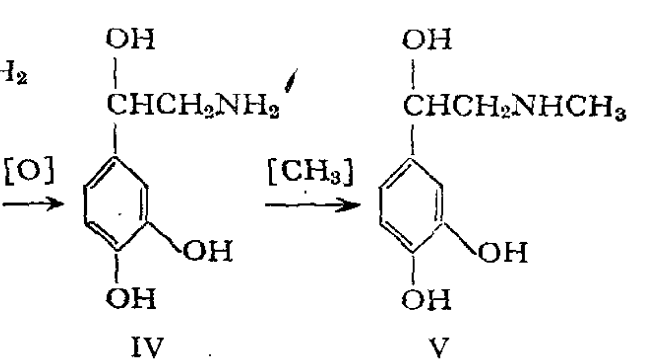

IV
图 1 儿茶酚胺生物合成主要途径 
由酪氨酸 (I) 到腎上腺素 (V) 的整个反应鏈 中，儿茶酚丙氨酸 (II) 在儿茶酚丙氨酸脫羧 酶参予下脫羧生成儿茶酚乙胺(III)的反应速 度較快 ${ }^{[2,3]}$; III 的側鏈 $\beta$ 位导入垹基，形成 去甲腎上腺索 (IV) 的酶, 是儿茶酚乙胺 $\beta$ 垟 化酶，这一步是鏈式反应中最慢的 ${ }^{[4,5]}$ 。催化 $\mathrm{IV}$,生成 $\mathrm{V}$ 的嘸，是去甲腎上腺素轉甲基酶。 上面各步反应中 II 的脫羧和 IV 的甲基化过 程知道得比較清楚, 而 III 的側鏈䍩基化变成 IV 这一步, 目前知道得不多。儿茶酚丙氨酸 脫羒酶还参予其它芳香族氨基酸，例 5-垟色 氨酸、苯丙氨酸等的脫羧反应; 儿茶酚乙胺 $\beta$ 垟化酶的专属性也不高，它亦能使苯乙胺 和酪胺側鏈挳基化。

除了上面的主要途径，人們亦开始注意 到存在其它支路的可能性。例如 I 的側鏈垟 基化, 生成对䍩基苯絲氨酸, 脫羧产生鱆胺, 后者再轉化成 IV; 或 II 的側鎹先行羥基化， 生成儿茶酚絲氨酸，后者脫羧变成 $I V^{[6]}$ 。

腎上腺素和去甲腎上腺素自交感神經末 梢释放，經与受体結合产生一系列效应后， 除少量直接与菊萄糖醛酸或硫酸生成酯，或

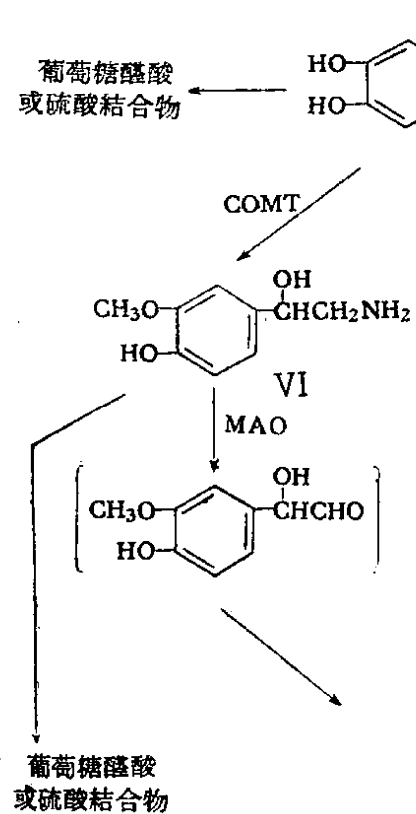

或硫㱆秸合物
以游离形式从尿中排出外, 它們在体內主要 通过氧化脫氨和酚垟基甲基化两条途径而失 活(图 2$)^{[7,8]}$ 。氧化脫氨过程需单胺氧化酶 (MAO) 参加。 IV 和 $V$ 脫氨后生成 3,4-二垟 基扁桃醛，后者在醛脫氫酶催化下迅速氧化 成 3,4-二垟基扁桃酸 (VIII)，再經儿茶酚氧 甲基轉移酶 (COMT) 作用，生成 3-甲氧基4-垟基扁桃酸(IX)。IV、V 酚垟基的甲基化需 儿茶酸氧甲基轉移酸参予。甲基化后生成的 間甲去甲掔上腺素(VI)和間甲腎上腺素(VII) 的活性很低，它們通过单胺氧化酶催化，最 后也变成 IX。究竟这两条代謝途径何者为 主？以前缺之足够的实驗資料，各人說法不 一。近来多数人訩为, 在形成和儲藏儿茶酚 胺的組織內, 它們是先通过单胺氧化酶的; 而循环內的儿茶酚胺，則先通过儿茶酚氧甲 基韩移酶 ${ }^{[9,10]}$ 。

体內游离的儿茶酚胺除了經过上述途 径，还可由于和儲存組織的再度結合而使活 性消失。

儿茶酚胺在体內合成后，便以結合的状 态儲存起来，当交感神經兴奋时，它自末梢 释放，作用于受体，产生 升压等一系列生理效应， 最后經代謝或与儲存組織 的再度結合而失活 (图 3)。 能阻断这一反应系列中任 何一步的化合物似有可能 产生降压作用 ${ }^{[11]}$ 。由于体 內生化反应的复杂性以及 各种支路的存在，每步反 应速度相差悬殊，要最終 确定一个葯物阻断那一个 ·或那几个环节, 往往是一 件繁重的工作。 


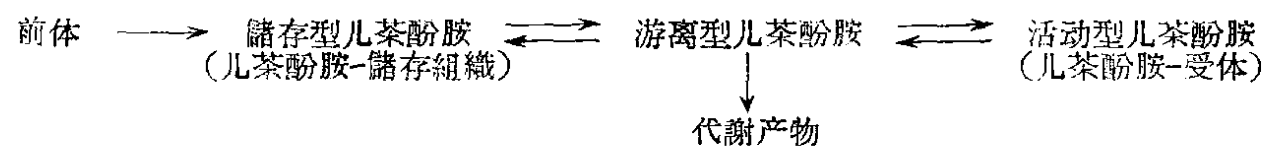

图 3 儿菜酚胺体內轉运途径

根据最近資料，阻滞由前体合成儿茶酚 胺的䒺物有儿茶酚丙氨酸脫羧酶抑制剂和儿 茶酚乙胺 $\beta$ 垟化酶抑制剂; 影响儲存的䒺物 有利血本、胍乙啶; 阻碍释放的有澳茮乙銨; 阻断儿茶酚胺与受体結合的有各种抗腎上腺 素䒺物(双茮胺、茮唑啉、哌年生、麦角毒等)。

\section{二 阻滞儿茶酚胺合成的綺物}

L- $\boldsymbol{\alpha}$-甲基儿茶酚丙氮酸 (Aldomet, $\mathrm{L}-\alpha-$ Methyldopa,X) ${ }^{[12,13]}$

口服有效，生效迅速，也能降低臥位血 压，但对严重及恶性高血压效果不好，作用 維持时間䄪 8-12 小时。D型异构体无效。 因为 $\mathrm{X}$ 在化学結构上与儿茶酚丙氨酸 (II) 相 似，过去訜为其作用机制在于竞孚性地抑制 了 II 的脫羌发，阻滞了去甲腎上腺素(IV)的生 成，从而降低了血压。但有人証明儿茶酚胺 生物合成中，II 脫羧变成儿茶酚乙胺(III)的 反应速度較快，郎使应用強烈的脫䍩酶抑制 剂，使 $75 \%$ 的酶被抑制，但对去甲腎上腺素 的生成仍无甚影响 ${ }^{[3,11]}$ 。最近 Sletzinger 等 ${ }^{[14]}$ 合成了X的两个类似物 $-\mathrm{DL}-\alpha-$ 甲基儿茶

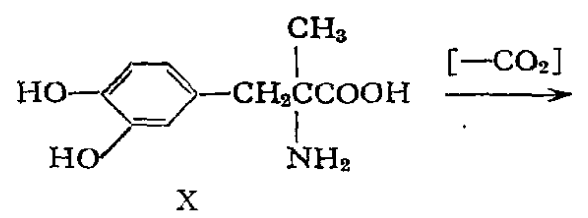<smiles>CC(N)Cc1ccc(O)c(O)c1</smiles>

$\cdot \mathrm{XI}$

酚丙肼酸 (XII) 和 DL- $a$-甲基儿茶酚丙垟酸 (XIII),发現均有強力的脱着酶抑制作用; 特 別是.XII，体外試驗效力比 X 約高一千倍，
但对高血压及正常血压的大鼠均沟有降压作 用。由此更証明了X的降压作用不是由于抑 制儿茶酚丙氨酸脱羧酶而产生的。

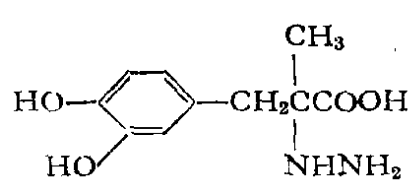

XII<smiles>CC(O)(Cc1ccc(O)c(O)c1)C(=O)O</smiles>

XIII

L- $a$-甲基儿茶酚丙 氨酸的降压机制現 有两种解释：一种訩为它在体內脱羧生成 $\alpha-$ 甲基儿茶酚乙朌 $(X I)$ ，后者在化学結构与䒺 理作用上与胍乙啶类似物 $\beta$-苯乙胍(XXXII) 相似，通过耗竭体內儿茶酚胺儲存量而使血 压下降 ${ }^{[15]}$; 另一种誩为脫羧生成的 XI 也可 进入去甲腎上腺素的儲存部位，神經兴奋时， 它作为伪神經激素释放出来，从而減弱了交 感神經兴奋強度，使血压下降 ${ }^{[16]}$ 。

\section{儿茶酚乙胺 $\beta$ 垟化酶抑制剂}

新近报导 NSD 1034（XIV)、NSD 1024 $(\mathrm{XV})$ 和 NSD 1055 (XVI) 能在体內和体外 抑制儿茶酚乙胺 $\beta$ 垟化酶。小鼠使用单胺氧 化酶抑制剂后引起的脑內去甲腎上腺素的壖 加，可被 NSD 1034 完全抑制之䄈。

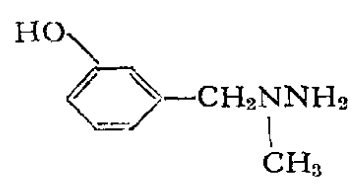

XIV

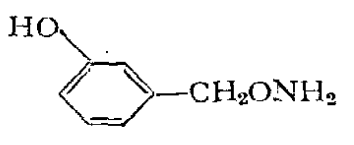

XV

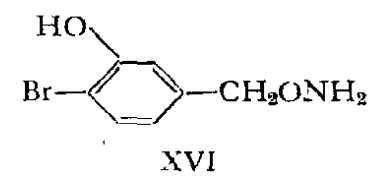




\section{三 影响儿茶酚胺僻存和释放的葯物}

仅在五年前这方面工作还局限在利血本 和 2,6-二甲莱胆碱醚( TM 10) 两个葯物上。 而在溴茮乙銨和胍乙啶間世后，影响儿茶酸 胺儲存和释放的䓎物立刻吸引了葯理学家、 生化学家和䒺化学家的注意力。在短期內合 成了大量結构类似物，进行了哚入的药理㸴 究，初步閭明了这些降压䒺物的作用机制。 目前它們不但成了监床上广为应用的新型降 压䒺，而且也用作药理分析工具，解决了交 感神經生化䒺理中許多舊有兴味的理論和实 际問題。在临床上用于降低血压的这类菞物 見表 $1^{[18]}$ 。

濞苇乙銨 (Bretylium, Darenthin, XVII) 它阻止交感神經末梢释放去甲腎上腺素

表 1 影响儿茶酚胺储存和释放的降压药物

\begin{tabular}{|c|c|}
\hline 䕀 物 名 称 & 鹙理作用及其祅制. \\
\hline $\begin{array}{l}\text { 2,6-二甲萫胆碱梄 } \\
\text { (TM 19) }\end{array}$ & 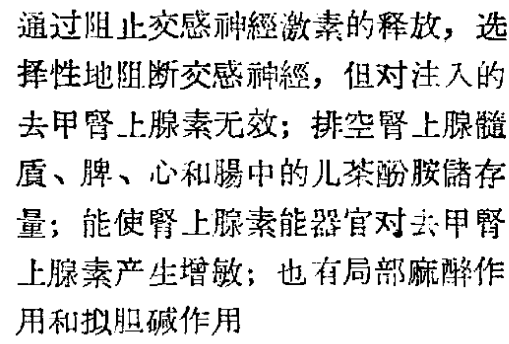 \\
\hline $\begin{array}{l}\text { 澳芥乙銨 } \\
\text { (Bretylium) }\end{array}$ & 像 TM10 \\
\hline $\begin{array}{l}\text { 利血平 } \\
\text { (Reserpine) }\end{array}$ & 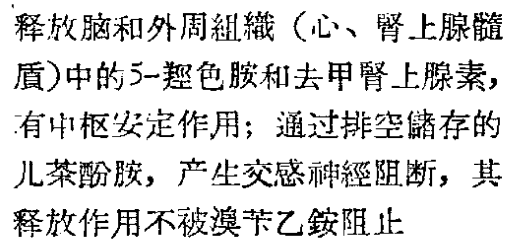 \\
\hline $\begin{array}{l}\text { 昔罗本 } \\
\text { (Syrosingopine) }\end{array}$ & $\begin{array}{l}\text { 与利血平相似，但中枢安定作用大 } \\
\text { 大減弱 }\end{array}$ \\
\hline $\begin{array}{l}\text { 胍乙啶 } \\
\text { (Guanethidine) }\end{array}$ & 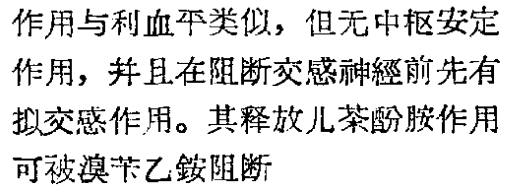 \\
\hline
\end{tabular}

而使血压下降，因此不影响副交感神經计动 的传递,沒有神經节阻滞剂共有的瞳孔放大、 便秘和口干等副作用。䒺效約維持六小时， 口服吸收不規勋, 能产生耐䓎性, 副作用有 头孺、腹泻、鼻塞及体位性低血压 ${ }^{[11]}$ 。

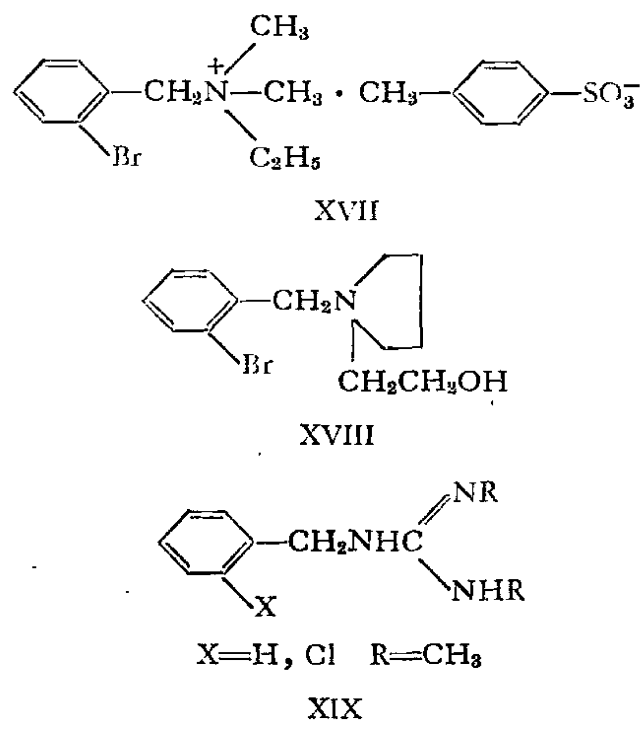

通过大量澳贲乙銨类似物的研究，其結 构与活性間的关系可从三方面来討論：（1） 季銨氮原子上的取代烷基可以是直鏈或环状 的，但不能太小或过大。如系三个甲基就沟 有作用，大于一个乙基、二个甲基时作用也 減弱 ${ }^{[19]}$ 。环状四亚甲基取代物 (XVIII) 仍有 效，但环护大至七元和八元时就无效 ${ }^{[20]}$ 。季 銨基被胍基置換后仍保留原有作用，胍基氮 上有甲基取代时 (XIX)活性增加 ${ }^{[21]}$ 。这可能 是強碱性胍基在体內与稹子結合，使氮原子 仍带正电荷，同样可以和受体負电荷中心結 合之故。（2）側鏈碳原子增至两个时虽有降 压作用，但作用机制已不是阻止儿茶酚胺的 释放，而是胍乙啶样的排空儿茶酚胺的存 量 ${ }^{[22]}$ 。（3）苯环邻位也可由氟、氯、碘、 甲基、硝基等取代而保留活性。澳換成橅后 产生了拟胆碱作用 ${ }^{[19]}$ 。

澳茮乙銨是一季銨化合物，在化学結构 上似应与乙酰胆碱更为接近。Burn ${ }^{[23,24]}$ 䚯 
为, 腎上腺素能神經內有胆碱能䊹維, 前者 兴奋时先释放出乙酰胆碱，乙酰胆碱作用于 去甲腎上腺素儲存部位，再継发性地释放出 去甲掔上腺素。澳苳乙銨由于对抗了乙酰胆 碱，从而阻抑了去甲堅上腺素的释放，广原生 交感神經阻断作用。

\section{胍乙啶 (Guanethidine, Ismelin, XXI)}

1957 年 Mull 等 ${ }^{[25]}$ 报告, 預先給予 3-N烑七环基丙偕胺肪 (CIBA 4092-Su, XX) 的 狗再給予苯丙胺或麻黃碱时，不引起血压升 高。 XX 也能降低神經型和腎型高血压动物 的血压，但对正常动物无作用。它生效緩慢， 作用可維持 2-一 $\mathrm{XX}$ 的类似物，但它仍是作用最強的，由于

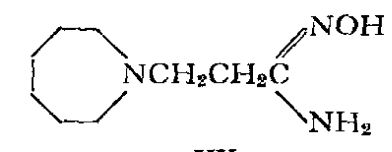

$\mathrm{XX}$<smiles>NC(N)CCN1CCCCCC1</smiles>

XXI

升高体温等副作用而未能用于临床。在浔找 副作用比 $\mathrm{XX}$ 小、降压效能更为满意的类似 物时，找到了胍乙啶(XXI)。

XXI 是一选择性高的交感神經阻滞剂。 ・它通过不断地释放来耗竭体內儿茶酚胺儲存 量，当神經兴奋时不再分泌足量的介稹使受 体兴奋，从而产生了交感神經化学解除。此 外，它的降压作用也可能与交感神經反射弧 的传入段或中枢段功能的改变和对血管本滑 肌的作用有关 ${ }^{[26]}$ 。胍乙啶能消除苯丙胺的加 压反应，但对去甲腎上腺素却产生增敏作用。 降压作用生效較慢，有效时間頗长，一次注 射能維持 6--9 天；因不阻断神經节，沟有 曈孔放大、口干和便秘等副作用，耐䒺性晌 不多見，监床評价高于溴菻乙銨 ${ }^{[11]}$ 。与利血
本不同，它不释放脑中去甲篮上腺素和 5-垟 色胺，也不易通过血脑屏障，因此无安定作 用。副作用有腹泻、鼻塞、体液滞留、心动 过緩和直立性低血压等。

胍乙啶出現后引起了人們广泛注意，在 樑入探明其䒺理机制和大量合成它的結构类 似物方面进行了巨量的工作。下面我們对 $\mathrm{XX}$ 和 XXI 的結构与活性关系作一較詳細的 討論 ${ }^{[27]}$ 。它們的結构可分成三部分：（1 ）氮 环；（2）側鏈；（3）含氮碱性基团。

（1）氮环它可被多种多样其它环取 代而仍保留活性。在含一个氮原子的环中, 只有七元和八元环的化合物具有满意的降压 作用，小于六元或大于九元环时活性很快減 弱或消失 ${ }^{[28,29]}$ 。氮五环和氮六环只有当它 們与另一环㪶合时，才有显著活性（XXII、 XXIII）。环上有两个氮原子时，在偕胺肪系 列化合物中失去活性; 在胍基系列化合物中 一般保留活性, 1,4-二氮六环化合物 (XXIV) 的活性比 1,4-二氮七环 $(X X V)$ 和 $1,5-$ 二氮

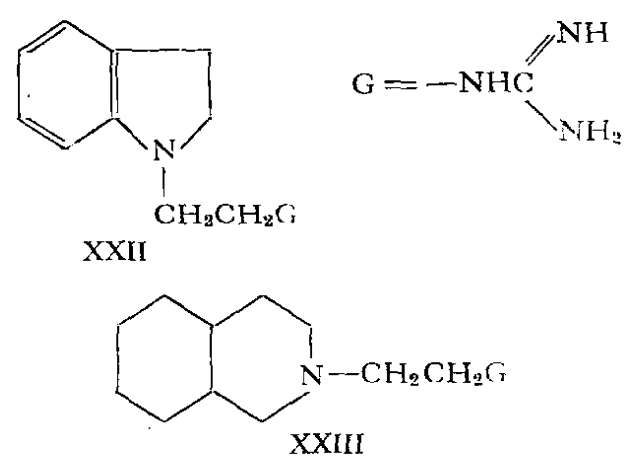

八环 (XXVI) 的高 ${ }^{[30]}$ 。环上烷基取代对作用 影响不大，例 XXIV $\left(\mathrm{R}^{\prime}=\mathrm{CH}_{3}\right)$ 和 XXVII 仍 有活性，但比未取代的差。

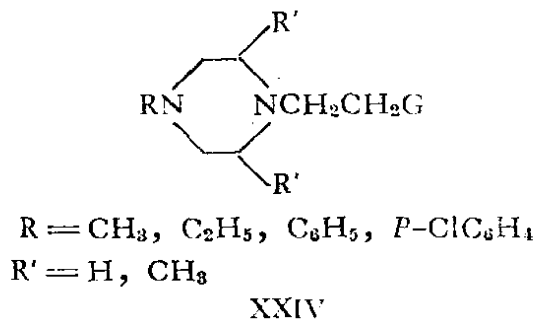




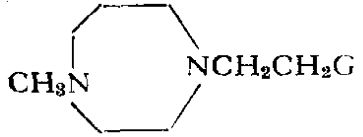

$\mathrm{XXV}$

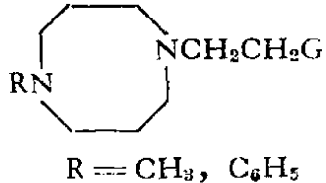

$\mathrm{XXVI}$
环上氮原子季銨化后(XXVIII、XXIX)活 性下降。氮环变成內酰胺环則活性消失 ${ }^{[3.17}$ 。

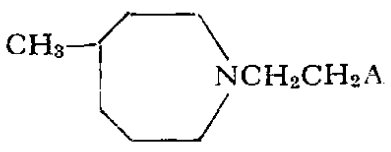

XXVII

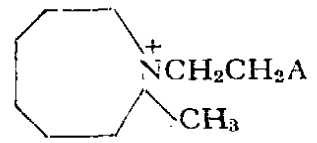

XXVIII

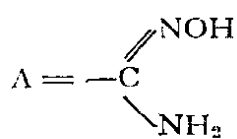

XXIX

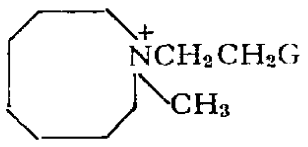

含氮桥环和稠环代替氮单环的化合物， 在偕胺肪系列中失去活性，而在胍基系列 (XXX) 中有时作用反增強 ${ }^{[27,31]}$ 。
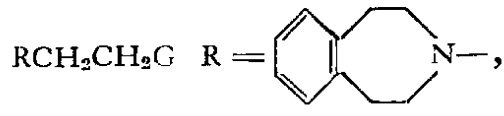

$\mathrm{xxx}$
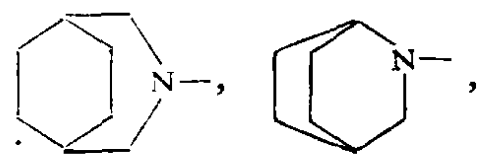

（高度活性）

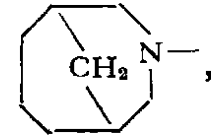

（高度活性）
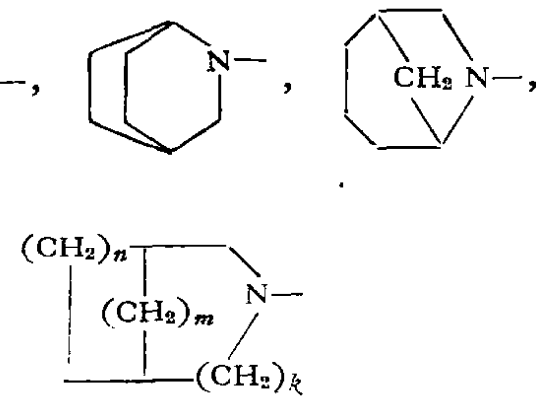

氮环被其它杂环如1，4-氧氮六环、1，4硫氮七环、噍噻嗪、咔唑等取代后, 均失去 活性 ${ }^{[28,29]}$ 。氮环打开，郎用直鏈烷基取代的 氨基代替氮环，导致作用減弱或消失，但二 乙氨基化合物 $\left(X X X ~ R=\left(\mathrm{C}_{2} \mathrm{H}_{5}\right)_{2} \mathrm{~N}-\right)$ 仍有良 好作用 ${ }^{[32]}$ 。

側鏈不与环上氮原子而与碳原子相連的 化合物 (XXXI)，有时仍能保持活性。最近 Costr: 等 ${ }^{[33]}$ 发現, 甚至用苯环代替氮杂环
（XXXII）时仍有很好的降压作用。

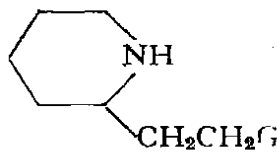

XXXI

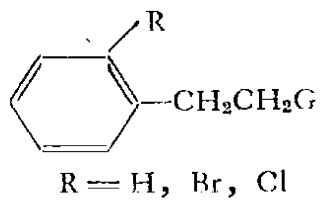

XXXII
（2）側鋪 只有当环与碱性基团間的 側鏈是两个未被取代的碳原子时才表現出活 性。任何长度的改变、側鏈上的取代, 均导

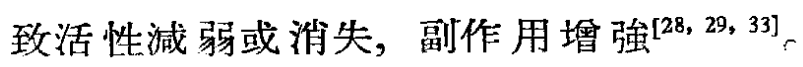
XXXII 的側鏈为一个碳原子时，作用轉为溴 芐乙銨型 ${ }^{[33]}$ 。

（3）含氮碱性基团 偕胺肜基的諩基 氢原子被酰基（XXXIII）或烷基取代时活性 大大降低或消失。胍基氮上的氫由次乙基 取代形成咪唑环（XXXIV）后，仍保留些降 压作用 ${ }^{[34]}$, 被硝基、酰 基 $\left(\mathrm{XXXV}_{\mathrm{a}}\right)$ 、氨基 $\left(\mathrm{XXXV}_{\mathrm{b}}\right)$ 、甲基或另一个胍基取代的衍生 物，活性下降或消失 ${ }^{[27,32,33,35]} \mathrm{c}$
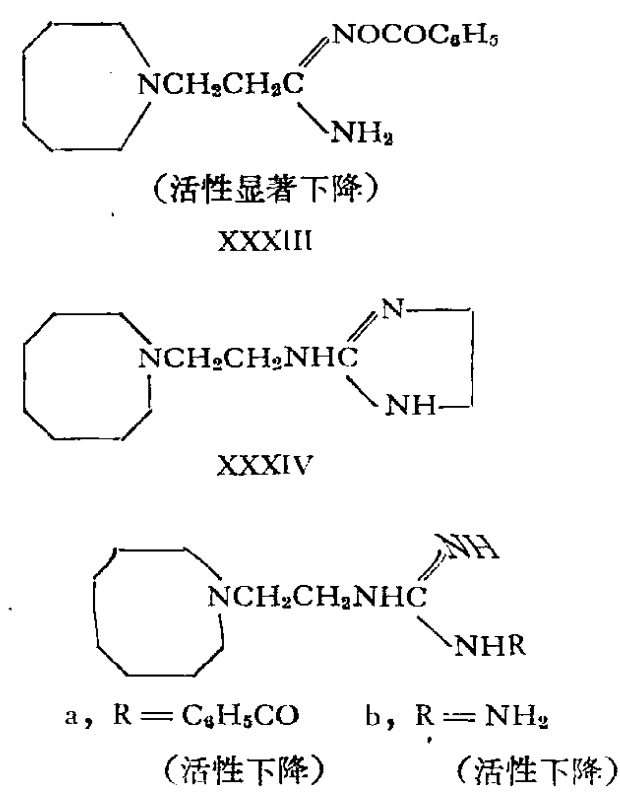

$\mathrm{XXXV}$

碱性基团換成脒基时仍有強大的降压作 用，例如 XXXVI 和 XXXVII，开环化合物没 有活性 ${ }^{[36,37]}$ 。換成异硫豚基、肼基、氨基、 取代氨基、氮七环基和氮八环基 ${ }^{[20]}$ 均无效。 


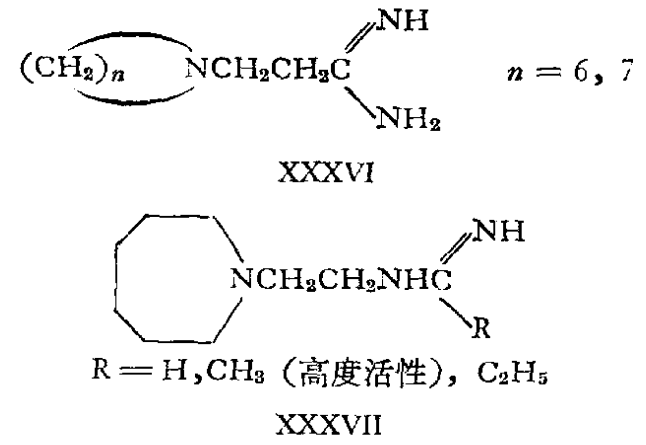

从上面化学結构与萂理作用关系的討論 中, 我們可以得出几点結論: 第一, 胍乙啶 类萂物的結构专属性不高, 头端氮环和尾端 含氮碱性基团部分都允海在結构上作較大的 变动; 但是第二，头端必需是一个环，尾端 必需是一在体內能与稹子結合、带上正电荷 的含氮碱性基团，作为側鏈的次乙基不能有 任何改变; 因此第三，胍乙啶类药物存在着 明显的基本結构 (XXXVIII)，它与儿茶酸胺 及拟交感神經䒺物的基本結构 (XXXIX) 頗 相似。也許它們在基本結构上的类似，正是 我們在㸴究胍乙啶释放儿茶酚胺的机理、体 內儲存儿茶酚胺的結合形式以及繁上腺素能 受体时，所应該注意的地方。

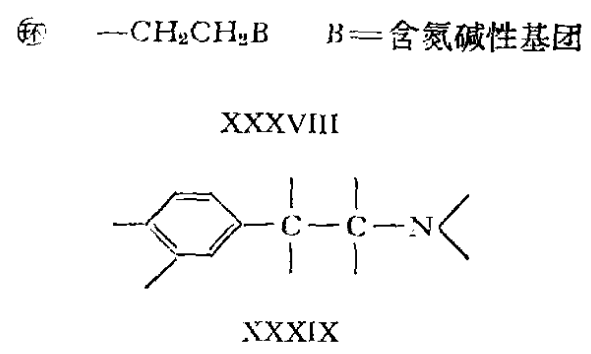

\section{四 腎上腺素能受体}

生物学过程常涉及三度空間。在抗体一 抗原、底物一酶和葯物一受体的研究中，証明 了生物体內这些生化反应都对分子构型、构 象、电子密度、酸碱性和溶解度等有严格的 要求。目前关于各种葯物受体的知識, 主要 是风分析䒺物分子的結构和所产生的生理、
药理作用中推导出来的, 至于萂物与受体作 用时的鱼实过渡态(它与底物一酶的米氏絡合 物不同)以及受体的精細結构則所知甚少 ${ }^{[38]}$ 。

交感神經兴奋时由末梢释放的去甲腎上 腺菜，通过怎样的机制使效应器产生反它呢? 这是一个重要而有趣的問題。人們提出了各 种假說，如用去甲腎上腺素本身的化学作用 和代謝的关系, 或用它引起組織中一系列电 活动来解释。但目前被大家接受利广泛应用 的是受体学說。

Ahlquist 訩为效应器中存在 $\alpha$ 与 $\beta$ 晒种 粲上腺素能受体(Adrenotropic receptor, Adrenergic receptor)。 $a$ 受体与各种兴奋現象有 关, 如使血管收縮、子宮和瞬膜兴奋, 但也 抑制腸平滑肌; $\beta$ 受体除产生心脏兴奋外主 要表現抑制作用，如使血管、子宮及支气管 平滑肌舒张。两种受体在各种組織和器官中 的分布是不均衡的 $\left.{ }^{[39}, 40\right]$ 。去甲腎上腺素只作 用于 $\boldsymbol{a}$ 受体，引起血压升高，而腎上腺素使 两种受体同时兴奋，其总的效果亦使血压升 高。麦角毒、双葉胺、哌冓生和葉唑啉等抗 腎上腺素䒺物仅阻断 $\boldsymbol{a}$ 受体，因而能使腎上 腺素升压作用出現反轉現象; 但对去甲腎上 腺素只減弱其升压作用,升不出現反轉現象。 3,4 -二氮异丙基腎上腺素 $(\mathrm{DCl})$ 只阳䉼 $\beta$ 受 体，故有阻断异丙基腎上腺素扩张支气管本 滑肌的作用。甲氧基非那明和双贸麦角胺能 同时阻断 $\alpha 、 \beta$ 受体 ${ }^{[41]}$ 。Furchgott 将受体分 为四种: $\boldsymbol{a}$ 受体引起平滑肌收縮， $\beta$ 使腸道 以外的本滑肌舒张、心搏頻率增加、心縮力 量加強， $\gamma$ 使糖原分解， $\delta$ 抑制腸道本滑 肌 ${ }^{[42,43]}$ 。目前多数学者主张将腎上腺素能受 体分成 $\alpha 、 \beta$ 两种。

近年来抗腎上腺素䒺物和拟交感䒺物在 䒺理和化学力面都得到了詳尽的㸴究, 积祭 
了詐多有关腎.上腺菜能受体生物化学概貌的 新的有价值的資料。我們現在結合自己的看 法, 对这方面的进展, 主要是 Belleau 等 ${ }^{[4]}$ 的 工作：做一簡略的介紹。

腎上腺素和去甲腎上腺素使腎上腺素能 受体兴奋是通过解酶与底物先生成絡合物， 再引起底物的化学变化产生的呢? 还是只通 过分子間庫军静电引力、范德华引力和氢鍵 的形成发生的? 是否受体本身就是酶, 有催 化儿茶酚胺氧化和氧甲基化的能力? 根据 $a, a-$ - 刖基苯乙胺仍具有兴奋效应, 和 $a$ 碳原子经用重氞标記的 (一) 去甲腎上腺素与 未标記的化合物对猫瞬膜的作用完全相们的 实驗結果，証明了受体不具有酶的特征，去 甲腎上腺素不是受体的底物。它們只通过靜 电抲力和范德华引力互相結合而至生兴奋。 儿茶湓胺和拟交感胺在体內生理 $\mathrm{pH}$ 条件 下，氮基与稹子結合带有正电荷; 腎上腺素 中的 $\mathrm{NH}$ 基团換成同电异素的氮原子时，由 于后者不生成带正电荷的鉡离子，故沒有活 性。因此儿茶酚胺的阳离子头端与受体負电 荷区域的結合, 是引起受体兴奋的主要条件。 靜电力与距离平方成反比。小的阳离子头端 有强的兴奋效应, 但当氨基上有烷基，如异 丙基肐代后，阳离子头端与受体負电荷中心 間引力大大減弱，受体兴奋效应降低，开始 出現了抑制作用。这一抑制作用可以誩为与

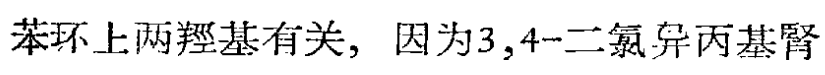
上腺素能阻䉼这一抑制效应。苯环上的垟基 通过形成金属 (如 $\mathrm{Fe}^{++[4]} 、 \mathrm{Mg}^{++}$等) 鳌合 物，与受体另一部分結合。抗腎上腺素䒺物 双苠胺的作用可分为两个阶段: 先是竞争 相, 随后出現非竞爭相。这可以这样解释: 双苳胺在体內先生成乙烯亚銨阳离子, 經过 折迭形成苯乙胺基本結构（图 4, a、b)，通过
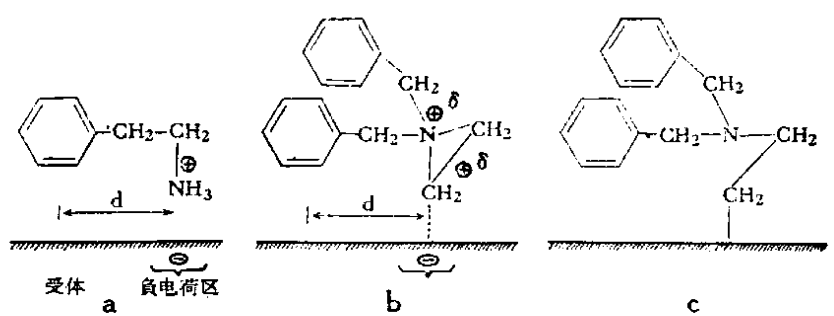

图 4 双苠胺对腎上腺素能受体的作用

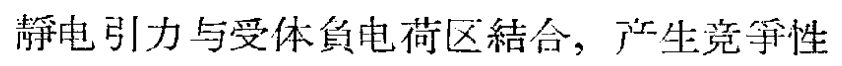
阻断; 然后进一步发生分子一重排, 生成碳阳 离子使受体酯化(图 4, c), 出現了非竞笋相。 三級胺能催化酯的水解, 被阴䉼受体的重生 吅能通过双茮胶分子内氮基对酯基发生分子 內自催化作用, 使后者水解来完成。 $\beta$-氨基 醇的羒酸酷很易水解，而磷酸酯比䡆稳定， 间时儲存的儿茶酚胺已被証明是与三磷酸腺 菖中的磷酸根以离子鍵互相結合 ${ }^{[40]}$, 因此受 体負电荷区似为磷酸根。这时被抗腎上腺素 紷物所阻断的受体的重生, 也可能通过休內 厂泛分布的磷酸酶的催化水解来实現。儿茶 酚膑和拟交感胺必需有莱环, 这䜅明受体对 芳香环有亲和力。图 5 为腎上腺素能受体示 意图 ${ }^{[47]}$ 。

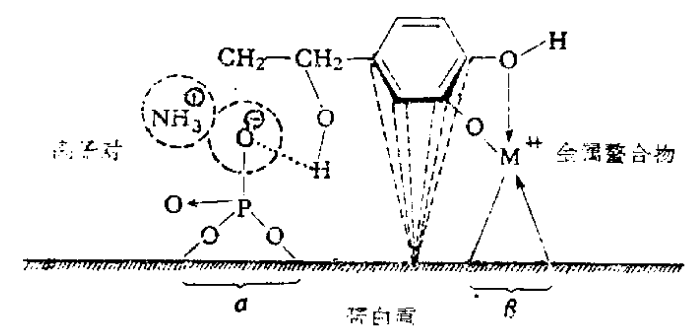

图 5 粲上腺素能受体(Belleau)

$\alpha$ 和 $\beta$ 两种受体是在同一实体的不同部 位, 这一实体可能是带有磷酸根的蛋白貭或 有一带磷酸根輔基的酶。 $\alpha$ 受体与此磷酸根 有关， $\beta$ 受体与 $\boldsymbol{a}$ 附近的蛋白貭某一区域有 关。 $\alpha 、 \beta$ 的兴奋均以离子对的形成为先决 条件。儿茶酚胺阳离子头端与受体磷酸根氧 阴离子間經过靜电引力形成离子鍵时， $\alpha$ 兴 
奋; 苯环垟基通过金属鳌合物的形成与受体 $\beta$ 部位連接，使 $\beta$ 兴奋; 側鏈垟基可以与受 体形成氫鍵,有利于儿茶酚胺与受体的結合; 苯环通过范德华引力与受体結合。

根据上面受体示噫图和对它的几点說 明，我們可以用来解释儿茶酚胺和大多数 拟交感神經葯物及受体阻断䒺物的作用类 型 ${ }^{[48-51]}$ 。例去甲腎上腺素有一小的阳离子头 端，电荷集中，能与 $\boldsymbol{\alpha}$ 受体产生最紧密的結 合，此时苯环上諩基虽能与 $\beta$ 結合，但它主 要表現为 $\boldsymbol{a}$ 兴奋效应。轺上腺素的氨基上取 代了甲基,使离子对間引力稍減弱, 因此 $\beta$ 兴 奋效应就同时表現出来了。异丙基腎上腺素 头端存在着异丙基的巨大空間障碍，使 $\boldsymbol{a}$ 兴 奋性娍弱, 因此主要表現为由苯环垹基引起 的 $\beta$ 兴奋效应。二氯异丙基整上腺素同样因 銨离子头端的立体障碍使 $\alpha$ 兴奋性显著下 降，但离子对間的引力仍能使分子在受体表 面得到定向排列; 可是此时苯环垟基已被氯 取代，无法与 $\beta$ 受体通过金属离子連接，故 它表現出 $\beta$ 受体阻断作用。二氯腎上腺素头 端已无空間障碍，因此除了表現出 $\beta$ 阻断作 用外，对 $\boldsymbol{a}$ 受体也有兴奋效应。

胍乙啶类化合物通过排空組織中儲存的 儿茶酚胺，使交感神經兴奋性全面下降。究 竟儲存的儿茶酚胺与組織如何結合，又通过 怎样的步驟被胍乙啶所释放呢? 这些問題我 們訩为可以应用上面有关受体的一些知識得 到解释。組織內与儿茶酚胺結合的部分可以 称为“儲存受体”，以与“效应受体”相区 別，它們的表面結构是相同的，但作为載体 的蛋白稹部分却不同，因此“儲存受体”与儿 茶酚胺結合时不会兴奋。这好象构造相同的 电子原件, 装在不同用途的电子仪器中。胍 乙啶类化合物基本結构与儿茶酚胺基本結构
頗相似。胍乙啶进入組織后与儿茶酚胺争夺 同一“儲存受体”并导致后者的释放。由于两 者对受体的亲和力相差不多，这一反应速度 較慢; 因此在葯理上就表現出胍乙啶排空組 織儿茶酚胺的速度不快，生效緩慢。上述假 說也可用于說明苯丙胺、酪胺等拟交感胺的 作用。它們在結构上与儿茶酚胺更相似, 通 过爭夺“儲存受体”来释放儿茶酚胺。

Belleau 对艄上腺素能受体結构的描述, 对儿茶酚胺与受体作用机制的假說，是在总 結前人积累的賀料和他自己工作的基础上所 作的新償試。虽然有許多矛盾和不完善的地 方, 份有待将来第一手材料来驗証, 但却是 生物受体学說継乙酰胆碱、鎮痛葯和組織胺 等受体之后的又一新进展。

\section{五結語}

由于菞化学家、萂理学家、生化学家等 的共同努力, 由于新技术新方法的广泛应用， 腎上腺素能神經䒺理在近年取得了很大进 展; 不但闈明了儿茶酚胺体內合成和代謝的 途径，而且从分子水平上探討了腎上腺素能 受体的結构和功能; 初步解决了作用于交感 神經的降压葯物的降压机制問題, 找到了影 响儿茶酚胺体內反应鏈上不同环节的降压葯 物; 更以此为䒺理分析工具，导致了㛑_上腺 素能神經新覌点的形成。所有这些已取得的 成果給了人們很大的鼓舞, 增強了継續前进 的信心。預計在今后十年中，借助于电子显 微鏡、电子計算机、 $\mathrm{X}$ 光衍射和核磁共振等 強大的技术手段，經过生物、化学和物理工 作者的密切配合，在揭露儿茶酚胺体內合成 和代謝的每一步細节以及受体三維結构方面 必将㢸更大的成績，从而为降压䒺物的設 計提供可靠的理論根据。为征服高血压病而 
战斗的人們正面临着一个㮫新的前景!

志謝: 承蒙稳汝运敎授果閱全文扭提供宝貴意

見，特此致謝。

[1] Blaschko, H., "Hypericnsion Recent Advances, The second Hahnemann symposium on hypertensive disease", P. 321, Henry Kimpton, London, 1962.

[2] Blaschko, H., ibid., P. 330.

[ 3 ] Brodie, B. B., Kuntzman, R., Hirsch, C. W., and Costa, E., Life Sci., 81 (1962).

[4] Holtz, P., Pharmacol. Rer., 11, 317 (1959).

[5] Clark, W. G., ibid., 11, 330 (1959).

[ 6 ] Kirshner, N., ibid., 11, 350 (1959).

[7] Gitlow, S. E., Mendelowitz, M., et al., "Hypertension Recent Advances", P. 335.

[8] Axelrod, J., "Ciba Foundation Symposium on Adrenergic Mechanisms", P. 28, J. \& A. Churchill, London, 1960.

[9] Zeller, E. A., "Hypertension Recent Advances", P. 342 .

[10] 张昌紹, “䒺理学进展” 第 1 頁, 上海科学技术出版 社, 1962。

[11] 甃汝运, 荺学学报, 10, 308 (1963)。

[12] Stone, C. A., Porter, C. C., Watson, L. S. and Ross, C. A., "Hypertension Recent Advance", P. 417.

[13] Brest, A. N., Seller, R., et al., ibid., P. 424.

[14] Sletzinger, M., Chemerda, J. M. and Bollinger, F. W., J. Med. Chem., 6, 101 (1963).

[15] Stone, C. A., Ross, C. A., Wenger, H. C., et al., J. Pharmacol., 136, 80 (1962).

[16] Day, M. D. and Rand, M. J., J. Pharm. Pharmacol., 15, 221 (1963).

[17] Kuntzman, R., Costa, E., et al., Life Sci., 85 (1962).

[18] Dipalma, J. R., "Hypertension Recent Advances", P. 393.

[19] Boura, A. L. A., Copp, F. C. and Green, A. F., Nature, 184, B. A. 70 (1959).

[20] 白东鲁、秒汝运, 化学学报, 待发表。

[21] Boura, A. L. A., Copp, F. C., Walton, E., et al., Nature, 191, 1312 (1961).

[22] Costa, E., Kuntzman, R., Gessa, G. L. and Brodie, B. B., Life Sci., 75 (1962).

[23] Burn, J. H., Brit. Med. J., 1, 1623 (1961).

[24] Burn, J. H. and Rand, M. J., "Advances in Pharmacology", vol. 1, P. 1, Academic Press, New
York, 1962.

[25] Mull, R. P., Maxwell, R. A. and Plummer, A. J., Nature, 180, 1200 (1957).

[26] Maxwell, R. A., "Hypertension Recent Advances", P. 437.

[27] Schlittler, E., Druey, J. and Marxer, A., "Fortschritte 'der Arzneimittelforschung", vol. 4, P. 295. Birkhäuser Verlag, Basel, 1962.

[28] Mull, R. P., Schmidt, P., Dapero, M. R., et al., J. Am. Chem. Soc., 80, 3769 (1958).

[29] Mull, R. P., Egbert, M. E. and Dapero, M. R., J. Org. Chem., 25, 1953 (1960).

[30] Mull, R. P., Mizzoni, R. H., et al., J. Med. Pharm. Chem., 5, 944 (1962).

[31] Najer, H., Giudicelli, R. et Sette, J., Bull. Soc. Chim. France, 1593 (1962).

[32] Short, J. H., Biermacher, U., Dunnigan, D. A. and Leth, T. D., J. Med. Chem., 6, 275 (1963).

[33] Costa, E., Kuntzman, R., Gessa, G. L. and Brodie, B. B., Life Sci., 75 (1962).

[34] Najer, H., Giudicelli, R. et Sette, J., Bull. Soc. Chem. France, 556 (1962).

[35] Najer, H., Giudicelli, R. et Sette, J., ibid., 559 (1962).

[36] Boura, A. L. A., Copp, F. C. and Green, A. . F., Nature, 195, 1213 (1962).

[37] Mull, R. P., Mizzoni, R. H., ct al., J. Med. Pharm. Chem., 5, 651 (1962).

[38] Beckett, A. H., 第一屆国际䒺理学会議交摘选录, 21 頁, 中国医学科学院情报研究室, 1962。

[39] Ahlquist, R. P., Am. J. Physiol., 153, 586 (19489,

[40] Folkow, B., "Ciba Foundation Syposium on Adrenergic Mechanisms", P. 190.

[41] Ahlquist, R. P., 第一屆国际䒺理学会議交摘选录, 30 頁。

[42] Furchgott, R. F., Pharm. Rev., 11, 429 (1959).

[43] Furchgott, R. F., "Ciba Foundation Syposium on Adrenergic Mechanisms" P. 246.

[44] Bclleau, B., ibid., P. 223.

[45] Imaizumi， R., 第一屆国际获理学会議文摘选录， 30 頁。

[46] Weiner, N., Pappas, P. and Jardetzky, O., ibicl., P. 27.

[47] Bloom, B. M. and Laubach, G. D., "Annual Review of Pharmacology", vol. 2, P. 95, Annual Reviews, Palo Alto, 1962.

[48] Ariöns, E. J., 第一屆国际荼理学会議文摘选录, 27 頁。

[49] Claassen, V., ibid., P. 29.

[50] Ariëns, E. J., "Ciba Foundation Syposium on Adrenergic Mechanisms", P. 253.

[51] Ariëns, E. J., ibid., P. 264. 